\title{
Стимулированное излучение лазерных структур InGaAs/GaAs/AIGaAs, выращенных методом газофазной эпитаксии из металлоорганических соединений на неотклоненной и отклоненной подложках $\mathrm{Ge} / \mathrm{Si}(001)$
}

\author{
(C) В.Я. Алешкин ${ }^{1,2}$, Н.В. Байдусь ${ }^{3}$, А.А. Дубинов ${ }^{1,2, \text { Ф З.Ф. Красильник }}{ }^{1,2}$, С.М. Некоркин ${ }^{1,3}$, \\ А.В. Новиков ${ }^{1,2}$, А.В. Рыков ${ }^{3}$, Д.В. Юрасов ${ }^{1,2}$, А.Н. Яблонский ${ }^{1}$ \\ ${ }^{1}$ Институт фризики микроструктур Российской академии наук, \\ 603950 Нижний Новгород, Россия \\ ${ }^{2}$ Нижегородский государственный университет им. Н.И. Лобачевского, \\ 603950 Нижний Новгород, Россия \\ ${ }^{3}$ Нижегородский научно-исследовательский институт \\ Нижегородского государственного университета им. Н.И. Лобачевского, \\ 603950 Нижний Новгород, Россия \\ E-mail: sanya@ipm.sci-nnov.ru
}

(Получена 16 ноября 2016 г. Принята к печати 21 ноября 2016 г.)

\begin{abstract}
Методом газофазной эпитаксии из металлоорганических соединений получены лазерные структуры $\mathrm{GaAs} / \mathrm{AlGaAs}$ с квантовыми ямами InGaAs на неотклоненной и отклоненной на $4^{\circ}$ к оси [011] подложках $\mathrm{Si}(001)$ с релаксированным буфером $\mathrm{Ge}$, излучающие в области прозрачности объемного кремния (длина волны больше 1100 нм при комнатной температуре). Пороговые плотности мощности наблюдения стимулированного излучения для структур, выращенных на неотклоненной и отклоненной подложках, составили 45 и $37 \mathrm{\kappa BT} / \mathrm{cm}^{2}$ соответственно.
\end{abstract}

DOI: 10.21883/FTP.2017.05.44431.8449

Переход от медных проводов к оптическим межсоединениям в высокопроизводительных процессорах может быть осуществлен с использованием гибридных $\mathrm{A}^{\mathrm{III}} \mathrm{B}^{\mathrm{V}}$-гетеролазеров, совместимых с комплементарной металлооксидной полупроводниковой технологией (CMOS) на кремнии [1]. Значительный прогресс был достигнут в создании лазеров на $\mathrm{Si}$-подложках с использованием гетероструктур $\mathrm{GaAs} / \mathrm{AlGaAs}$ с квантовыми ямами (КЯ) InGaAs [2-4], а также с квантовыми точками (КT) InAs [5,6]. Практически во всех случаях рост лазерных структур $\mathrm{A}^{\mathrm{III}} \mathrm{B}^{\mathrm{V}}$ был осуществлен на кремниевых подложках, отклоненных на несколько градусов от оси [001], чтобы избежать формирования антифазных дефектов на границе полярного и неполярного материалов [7]. В то же время существующие технологии изготовления кремниевых процессоров развиты для точно ориентированных подложек $\mathrm{Si}(001)$, с отклонением, не превышающим $0.5^{\circ}$ [8].

Недавно был достигнут успех в создании лазера GaAs/AlGaAs c 3 КЯ InGaAs, выращенного методом газофазной эпитаксии из металлоорганических соединений (МОГФЭ) на неотклоненой подложке $\mathrm{Si}(001)$ с буферным слоем Ge [9]. Лазер излучает на длине волны 992 нм при комнатной температуре. Однако для использования $\mathrm{A}^{\mathrm{III}} \mathrm{B}^{\mathrm{V}}$-лазеров в кремниевых оптических межсоединениях необходимо, чтобы длина волны $(\lambda)$ излучения лазера попадала в область прозрачности объемного кремния $(\lambda>1100$ нм при комнатной температуре). Отметим, что достичь этого диапазона длин волн удалось в лазерной структуре с двойными квантовыми ямами
InGaAs/GaAsSb/GaAs, выращенной на отклоненной подложке $\mathrm{Si}(001)$ с использованием релаксированного буфеpa Ge, но только при температуре жидкого азота [10].

В связи с этим в настоящей работе созданы и исследованы лазерные структуры GaAs/AlGaAs с одиночными КЯ InGaAs с бо́льшим по сравнению со структурой, исследованной в работе [9], содержанием индия для увеличения длины волны излучения. Лазерные структуры выращивались методом МОГФЭ на подложках $\mathrm{Si}(001)$ с использованием релаксированного буферного слоя $\mathrm{Ge}$. Для сравнения излучательных характеристик при оптической накачке лазерные структуры были выращены как на точно ориентированной (неотклоненной) подложке $\mathrm{Si}(001)$, так и на подложке, отклоненной на $4^{\circ}$ от оси [001] к оси [011].

Неотклоненная и отклоненная на $4^{\circ}$ к оси [011] виртуальные подложки $\mathrm{Ge} / \mathrm{Si}(001)$ были выращены в сверхвысоковакуумной установке молекулярно-пучковой эпитаксии (МПЭ) Riber SIVA-21 по методике так называемого „двухстадийного“ роста [11-13]. Температура роста контролировалась с помощью откалиброванной термопары [14] и специализированного инфракрасного пирометра IMPAC IS 12. Осаждение $\mathrm{Si}$ и Ge осуществлялось путем электронно-лучевого испарения. Контроль состояния поверхности в процессе эпитаксии осуществлялся методом дифракции быстрых электронов (ДБЭ). Морфология поверхности выращенных образцов исследовалась с помощью атомно-силовой микроскопии (ACM) на микроскопе NTEGRA Prima. Кристаллическое качество образцов исследовалось с помощью рентгено- 
дифракционного анализа, а также с помощью метода селективного травления дефектов [15].

Суть метода двухстадийного роста заключается в следующем. На первом этапе осаждается относительно тонкий $(\sim 50$ нм) слой Ge при пониженной температуре $\left(275^{\circ} \mathrm{C}\right)$, чтобы исключить релаксацию упругих напряжений через образование трехмерных островков. При этом релаксация происходит путем образования большого числа дислокаций несоответствия и образуется относительно гладкий $(\sim 1 \mathrm{Hм})$ слой с большой плотностью прорастающих дислокаций $\left(>10^{10} \mathrm{~cm}^{-2}\right)$. На втором этапе температура роста повышается до $600^{\circ} \mathrm{C}$ и основная часть слоя $\mathrm{Ge}(\sim 1$ мкм в настоящей работе) выращивается при данной температуре, что способствует повышению кристаллического качества. Для снижения плотности прорастающих дислокаций после формирования структуры применялся циклический отжиг: $\left\{850^{\circ} \mathrm{C} / 2\right.$ мин, $550^{\circ} \mathrm{C} / 2$ мин $\} \times 5$ повторений. Согласно результатам измерения с использованием метода АCM в силу достаточно короткого времени выдержки при высокой температуре удается сохранить малую шероховатость поверхности после цикла отжига (среднеквадратичная шероховатость RMS $<1$ нм на площади скана $30 \times 30$ мкм), при этом эффективно снижая плотность прорастающих дислокаций до значений $\sim(1-2) \cdot 10^{7} \mathrm{~cm}^{-2}$ (по подсчету ямок травления). Сравнение образцов со слоями $\mathrm{Ge}$ одинаковой толщины, выращенных на отклоненных и неотклоненных подложках $\mathrm{Si}$, не выявило существенных различий в значениях плотности прорастающих дислокаций. Кроме того, при выбранных условиях отжига (и толщине слоя Ge) процесс диффузии $\mathrm{Si}$ из подложки практически не затрагивает верхний, приповерхностный слой $\mathrm{Ge}$, на котором впоследствии осуществляется рост слоев $\mathrm{A}^{\mathrm{III}} \mathrm{B}^{\mathrm{V}}[16]$. Высокое качество получаемых виртуальных подложек $\mathrm{Ge} / \mathrm{Si}$ также подтверждается данными рентгеновской дифракции, согласно которым слои Ge характеризуются малой шириной кривой качания $\left(\sim 0.05^{\circ}\right)$.

На отклоненной и неотклоненной подложках $\mathrm{Ge} / \mathrm{Si}$ в установке МОГФЭ AIX 200RF при пониженном давлении (100 мбар) осуществлялся рост лазерных гетероструктур. В качестве источников основных элементов использовались триметилгаллий, триметилалюминий, триметилиндий и арсин. Перед началом роста проводился 5-минутный отжиг виртуальных подложек $\mathrm{Ge} / \mathrm{Si}$ в потоке водорода и арсина при $670^{\circ} \mathrm{C}$. При этой же температуре со скоростью $0.33 \mathrm{HM} / \mathrm{c}$ выращивался буферный слой, состоящий из слоев $\operatorname{AlAs}(10$ нм $) / \mathrm{GaAs}(50$ нм $) / \operatorname{AlAs}(10$ нм). Отношение элементов V и III групп для этих слоев составляло 30. Предварительные исследования показали, что такие буферные слои обеспечивают зеркальную поверхность, а в сигнале фотолюминесценции - отсутствие линий, связанных с комплексами германия [17]. Это обеспечивается встраиванием тонкого слоя AlAs на границе раздела $\mathrm{GaAs} / \mathrm{Ge}$, благодаря чему уменьшается взаимодиффузия атомов из-за высокой энергии связи $\mathrm{Al}-\mathrm{As}$ [18]. После
Параметры лазерных структур

\begin{tabular}{c|l|c}
\hline Номер слоя & \multicolumn{1}{|c|}{ Состав слоя } & Толщина, нм \\
\hline 1 & $\mathrm{AlAs}$ & 10 \\
2 & $\mathrm{GaAs}$ & 50 \\
3 & $\mathrm{AlAs}$ & 10 \\
4 & $\mathrm{GaAs}$ & 500 \\
5 & Градиент $\mathrm{GaAs} \rightarrow \mathrm{Al}_{0.3} \mathrm{Ga}_{0.7} \mathrm{As}$ & 100 \\
6 & $\mathrm{Al}_{0.3} \mathrm{Ga}_{0.7} \mathrm{As}$ & 1000 \\
7 & $\mathrm{GaAs}_{2}$ & 250 \\
8 & $\mathrm{In}_{x} \mathrm{Ga}_{1-x} \mathrm{As}$ & 10 \\
9 & $\mathrm{GaAs}_{10}$ & 20 \\
11 & $\mathrm{Al}_{0.3} \mathrm{Ga}_{0.7} \mathrm{As}$ & 10 \\
12 & $\Gamma_{2} \mathrm{Al}_{0.3} \mathrm{Ga}_{0.7} \mathrm{As} \rightarrow \mathrm{GaAs}$ & 10
\end{tabular}

слоя GaAs толщиной 500 нм выращивался ограничивающий слой AlGaAs при температуре $720^{\circ} \mathrm{C}$. Окружающие КЯ слои InGaAs выращивались при $620^{\circ} \mathrm{C}$. Отношение элементов V и III групп составляло 80. У поверхности располагался тонкий $(20 \mathrm{HM})$ слой $\mathrm{AlGaAs}$ для уменьшения влияния поверхностной рекомбинации, а также тонкий покровный слой GaAs для предотвращения окисления. Подробные параметры слоев лазерных структур приведены в таблице.

Оптические свойства выращенных структур исследовались на выколотых образцах шириной $\sim 10$ мм. Возбуждение стимулированного излучения осуществлялось излучением параметрического генератора света „Spectra-Physics“ MOPO-SL (длина волны излучения 760 нм, длительность импульса 5 нс, частота повторения импульсов 10 Гц). Накачивалась полоска размерами $10 \times 0.2$ мм. Для регистрации сигнала ФЛ использовался решеточный монохроматор Acton 2300i и многоканальный фотоприемник на основе линейки фотодиодов InGaAs (спектральный диапазон 600-2200 нм). Измерения проводились при комнатной температуре.

На рис. 1 представлены спектры стимулированного излучения трех лазерных структур. Из рисунка видно, что использование в структурах 2 и 3 КЯ с бо́льшим содержанием индия по сравнению со структурой 1 привело к существенному сдвигу линий излучения в длинноволновую область. Также видно, что, несмотря на одновременное выращивание частей $\mathrm{A}^{\mathrm{III}} \mathrm{B}^{\mathrm{V}}$ в структурах 2 и 3, положение линий стимулированного излучения в этих структурах существенно различается. Вероятно, различие связано с зависимостью встраивания In в КЯ InGaAs от ориентации подложки. Другой причиной может служить частичная релаксация напряжений в КЯ InGaAs в структуре, выращенной на неотклоненной подложке.

В структуре 2 длина волны стимулированного излучения составляет $\sim 1110$ нм, в структуре 3 длина волны стимулированного излучения $>1130$ нм. Отметим, что и в том, и в другом случае длина волны излучения лежит в области прозрачности объемного кремния, вследствие 


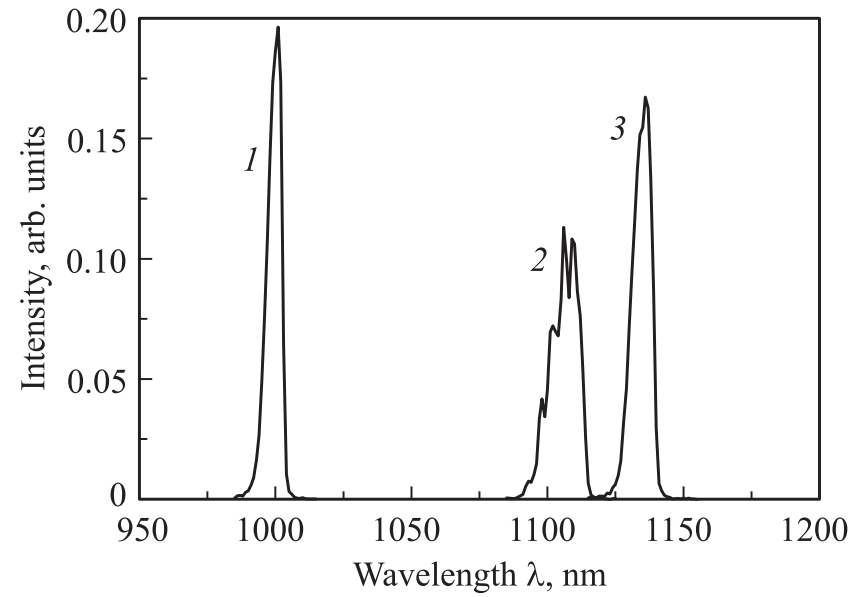

Рис. 1. Спектры стимулированного излучения лазерных структур: 1 - структура, использованная в работе [9] (доля In $x \approx 0.17), 2$ - структура на отклоненной подложке $\mathrm{Ge} / \mathrm{Si}$ $(x \approx 0.33), 3$ - структура на неотклоненной подложке $\mathrm{Ge} / \mathrm{Si}$ $(x \approx 0.35)$.

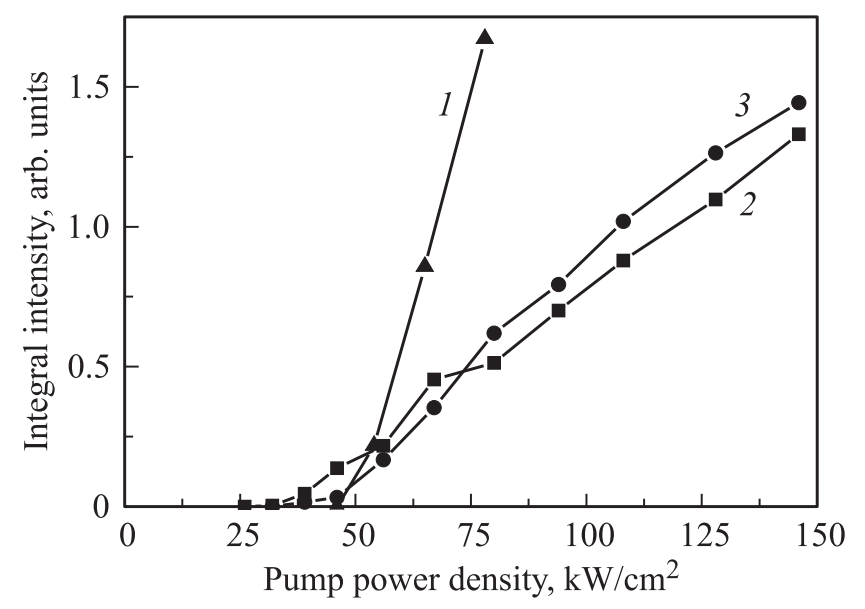

Рис. 2. Зависимости интегральной интенсивности стимулированного излучения лазерных структур: 1 - структура, исследованная в работе [9], 2 - структура на отклоненной подложке $\mathrm{Ge} / \mathrm{Si}, 3$ - структура на неотклоненной подложке $\mathrm{Ge} / \mathrm{Si}$.

чего эти источники оказываются пригодными для каналирования и распространения излучения с малыми потерями в кремниевых волноводах.

На рис. 2 представлены зависимости интегральной интенсивности стимулированного излучения трех лазерных структур от плотности мощности оптического возбуждения. Из измеренных зависимостей были получены значения пороговой плотности мощности возникновения стимулированного излучения: $\sim 37$ кВт/см ${ }^{2}$ для структуры на отклоненной подложке и $\sim 45 \mathrm{\kappa B} / \mathrm{cm}^{2}$ для структур на неотклоненной подложке. Отметим, что порог генерации при комнатной температуре для лазерного диода, изготовленного из структуры 1 , составлял $\sim 5.5$ кА/см ${ }^{2}[9]$; следовательно, аналогичные значе- ния пороговой плотности тока ожидаются при электрической накачке и для лазерных диодов, изготовленных из структур 2 и 3. Кроме того, видно, что использование буферного слоя, состоящего из чередующихся слоев AlAs и GaAs, существенно уменьшает количество дефектов и приводит к уменьшению пороговой плотности мощности в 2 раза по сравнению с лазерной структурой $\mathrm{A}^{\mathrm{III}} \mathrm{B}^{\mathrm{V}}$ на подложке $\mathrm{Ge} / \mathrm{Si}$, но без такого буфера [3]. Хотя порог генерации для структуры 2 ниже, чем для структуры 3, за порогом генерации интенсивность излучения структуры 3 выше, чем структуры 2, что говорит о большей квантовой эффективности структуры на неотклоненной подложке. Больший наклон зависимости интегральной интенсивности стимулированного излучения от плотности мощности накачки для структуры 1 на рис. 2, возможно, связан с бо́льшим числом КЯ (3 КЯ) в этой структуре по сравнению с остальными структурами (1 КЯ).

Таким образом, показано, что использование буферного слоя, состоящего из чередующихся слоев AlAs и GaAs, приводит к значительному уменьшению дефектов в лазерных структурах $\mathrm{A}^{\mathrm{III}} \mathrm{B}^{\mathrm{V}}$, выращенных как на неотклоненной, так и на отклоненной на $4^{\circ}$ к оси [011] подложках $\mathrm{Ge} / \mathrm{Si}(001)$. Это подтверждается уменьшением в 2 раза пороговой плотности мощности возбуждения. Формирование антифазных дефектов на неотклоненной подложке несущественно сказывается на излучательных свойствах. Полученные результаты свидетельствуют о возможности получения на точно ориентированных подложках $\mathrm{Ge} / \mathrm{Si}(001)$ низкопороговых гибридных лазеров, излучающих в области прозрачности объемного кремния.

Работа выполнена при финансовой поддержке РНФ (проект № 14-12-00644).

\section{Список литературы}

[1] D. Liang, A.W. Fang, J.E. Bowers. In: Fibre Optic Communication [Springer Series in Optical Sciences, Vol. 161], ed. by H. Venghaus, N. Grote (Springer-Verlag, Berlin/Heidelberg, 2012) p. 625.

[2] Y. Chriqui, G. Saint-Girons, S. Bouchoule, J.-M. Moison, G. Isella, H. von Kaenel, I. Sagnes. Electron. Lett., 39, 1658 (2003).

[3] В.Я. Алешкин, Н.В. Дикарева, А.А. Дубинов, С.А. Денисов, З.Ф. Красильник, К.Е. Кудрявцев, С.А. Матвеев, С.М. Некоркин, В.Г. Шенгуров. Письма ЖЭТФ, 100, 900 (2014).

[4] J. Wang, X. Ren, C. Deng, H. Hu, Y. He, Z. Cheng, H. Ma, Q. Wang, Y. Huang, X. Duan, X. Yan. J. Lightwave Technol., 33, 3163 (2015).

[5] A.Y. Liu, C. Zhang, J. Norman, A. Snyder, D. Lubyshev, J.M. Fastenau, A.W.K. Liu, A.C. Gossard, J.E. Bowers. Appl. Phys. Lett., 104, 041104 (2014).

[6] S. Chen, W. Li, J. Wu, Q. Jiang, M. Tang, S. Shutts, S.N. Elliott, A. Sobiesierski, A.J. Seeds, I. Ross, P.M. Smowton, H. Liu. Nature Photonics, 10, 307 (2016). 
[7] H. Kroemer, K.J. Polasko, S.C. Wright. Appl. Phys. Lett., 36, 763 (1980).

[8] K. Volz, A. Beyer, W. Witte, J. Ohlmann, I. Németh, B. Kunert, W. Stolz. J. Cryst. Growth, 315, 37 (2011).

[9] V.Ya. Aleshkin, N.V. Baidus, A.A. Dubinov, A.G. Fefelov, Z.F. Krasilnik, K.E. Kudryavtsev, S.M. Nekorkin, A.V. Novikov, D.A. Pavlov, I.V. Samartsev, E.V. Skorokhodov, M.V. Shaleev, A.A. Sushkov, A.N. Yablonskiy, P.A. Yunin, D.V. Yurasov. Appl. Phys. Lett., 109, 061111 (2016).

[10] А.Н. Яблонский, С.В. Морозов, Д.М. Гапонова, В.Я. Алешкин, В.Г. Шенгуров, Б.Н. Звонков, О.В. Вихрова, Н.В. Байдусь, З.Ф. Красильник. ФТП, 50, 1455 (2016).

[11] L. Colace, G. Masini, F. Galluzzi, G. Assanto, G. Capellini, L. Di Gaspare, E. Palange, F. Evangelisti. Appl. Phys. Lett., 72, 3175 (1998).

[12] H.-C. Luan, D.R. Lim, K.K. Lee, K.M. Chen, J.G. Sandland, K. Wada, L.C. Kimerling. Appl. Phys. Lett., 75, 2909 (1999).

[13] Д.В. Юрасов, А.И. Бобров, В.М. Данильцев, А.В. Новиков, Д.А. Павлов, Е.В. Скороходов, М.В. Шалеев, П.А. Юнин. ФТП, 49, 1463 (2015).

[14] П.В. Волков, А.В. Горюнов, А.Ю. Лукьянов, А.Д. Тертышник, А.В. Новиков, Д.В. Юрасов, Н.А. Байдакова, Н.Н. Михайлов, В.Г. Ремесник, В.Д. Кузьмин. ФТП, 46, 1505 (2012).

[15] L. Souriau, T. Atanasovac, V. Terzieva, A. Moussa, M. Cay$\max$, R. Loo, M. Meuris, W. Vandervorst. J. Electrochem. Soc., 155, H677 (2008).

[16] J.M. Hartmann, A. Abbadie, J.P. Barnes, J.M. Fedeli, T. Billon, L. Vivien. J. Cryst. Growth, 312, 532 (2010).

[17] V.Ya. Aleshkin, A.A. Dubinov, K.E. Kudryavtsev, V.V. Rumyantsev, A.A. Tonkikh, N.D. Zakharov, B.N. Zvonkov. J. Appl. Phys., 115, 043512 (2014).

[18] C.K. Chia, J.R. Dong, D.Z. Chi, A. Sridhara, A.S.W. Wong, M. Suryana, G.K. Dalapati, S.J. Chua, S.J. Lee. Appl. Phys. Lett., 92, 141905 (2008).

Редактор Л.В. Шаронова

\section{Stimulated emission \\ of InGaAs/GaAs/AIGaAs laser structures, grown by MOCVD on exact and offcut $\mathrm{Ge} / \mathrm{Si}(001)$ substrates}

V.Ya. Aleshkin ${ }^{1,2}$, N.V. Baidus ${ }^{3}$, A.A. Dubinov ${ }^{1,2}$, Z.F. Krasilnik ${ }^{1,2}$, S.M. Nekorkin ${ }^{1,3}$, A.V. Novikov ${ }^{1,2}$, A.V. Rykov ${ }^{3}$, D.V. Yurasov ${ }^{1,2}$, A.N. Yablonskiy ${ }^{1}$

${ }^{1}$ Institute for Physics of Microstructures, Russian Academy of Sciences, 603950 Nizhny Novgorod, Russia

${ }^{2}$ Lobachevsky State University of Nizhny Novgorod, 603950 Nizhny Novgorod, Russia

${ }^{3}$ Physical-Technical Research Institute of Lobachevsky State University of Nizhny Novgorod, 603950 Nizhny Novgorod, Russia

Abstract $\mathrm{GaAs} / \mathrm{AlGaAs}$ laser structures with InGaAs quantum well were obtained by metalloorganic chemical vapor deposition (MOCVD) on an exact and $4^{\circ}$ offcut $\mathrm{Si}(001)$ substrates with a Ge buffer layer. Laser structures radiated in transparency region of bulk silicon (wavelength is greater than $1100 \mathrm{~nm}$ at room temperature). The threshold power density for stimulated emission observing of structures grown on exact and offcut substrates accounted for 45 and $37 \mathrm{~kW} / \mathrm{cm}^{2}$, respectively. 